\title{
HDL Models of Ferromagnetic Core Hysteresis Using Timeless Discretisation of the Magnetic Slope
}

\author{
Hessa Al-Junaid and Tom Kazmierski \\ University of Southampton, UK \{hjaa01r, tjk\}@ecs.soton.ac.uk
}

\begin{abstract}
A new methodology is presented to assure numerically reliable integration of the magnetisation slope in the JilesAtherton model of ferromagnetic core hysteresis. Two HDL implementations of the technique are presented, one in SystemC and the other in VHDL-AMS. The new model uses timeless discretisation of the magnetisation slope equation and provides superior accuracy and numerical stability especially at the discontinuity points that occur in hysteresis. Numerical integration of the magnetisation slope is carried out by the model itself rather than by the underlying analogue solver. The robustness of the model is demonstrated by practical simulations of examples involving both major and minor hysteresis loops.
\end{abstract}

\section{Introduction}

Modeling and simulating a magnetic core based on the theory of ferromagnetic hysteresis is a process that involves many modeling and numerical difficulties due to the nonlinear nature of the hysteric and discontinuities in the magnetisation slope. Discontinuities occur at the turning points when the applied field changes its direction. This property of hysteresis is the cause of frequent numerical failures experienced by the analogue solver of the simulator used. The Jiles-Atherton (JA) model of ferromagnetic hysteresis [2] has been used extensively for creating non-linear models of magnetic materials in many commercial circuit simulators, such as SPICE and SABER, therefore JA model is used in this work. Practical implementation of the JA model is not straightforward as it involves numerical integration of a discontinuous and non-linear differential equation. In addition, the model in its original form can sometimes produce a hysteresis curve with negative slopes for which there is no physical justification [1]. Also, there have been claims that the JA model suffers from convergence problems and long analysis times.

The advent of Analogue and Mixed-Signal (AMS) Hardware Description Languages (HDLs) has created new op- portunities to develop enhanced and reliable models of difficult components. As AMS HDLs support modeling in mixed physical domains, they provide excellent environments for modeling the ferromagnetic hysteresis. The model proposed here overcomes the problem of numerical reliability by using a special, timeless discretisation technique to integrate the magnetisation slope $\frac{d M}{d H}$. Unlike most existing implementations, the new technique does not rely on time-based integration of $\frac{d M}{d H}$ and consequently does not involve the underlying analogue solver. Previously published HDL implementations of the JA model $[4,5]$ also suffer from occasional numerical stability as they rely on either differential or integral operators which are handled by the analogue solver. The model has been implemented in two HDLs: SystemC [3] and VHDL-AMS and both implementations produce virtually identical results.

\section{Timeless discretisation of the magnetisation slope}

The most important challenge in the implementation of the JA model is the calculation of the magnetisation slope given by Eq. 1 .

$$
\frac{d M}{d H}=\frac{1}{(1+c)} \frac{M_{a n}-M}{\frac{\delta k}{\mu_{0}}+\alpha\left(M_{a n}-M\right)}+\frac{c}{(1+c)} \frac{d M_{a n}}{d H}
$$

Eq. 1 is a nonlinear differential equation with incremental terms which needs to be solved but it cannot be solved directly by a simulator designed to calculate the time derivatives of unknown waveforms. Most implementations of the JA model require awkward conversion of the magnetisation derivative $\frac{d M}{d H}$ to time derivatives. This is usually implemented by calculating the derivative of $H$ with respect to time $\frac{d H}{d t}$, and then integrating $\frac{d M}{d t}$ (e.g. using the VHDLAMS 'INTEG operator). Our discretisation approach is more general as it uses an independent process. The process is responsible for directly performing timeless integration of $\frac{d M}{d H}$ using $H$ as the independent variable, not the time. This approach is more reliable and fast. We have implemented this using Forward Euler integration method. Our model is 
capable of producing minor loops with no numerical difficulties for various minor loops sizes and in different positions. Both SystemC and VHDL-AMS implementations are using the same parameters as in the original paper by Jiles and Atherton [2] except for $a 2$. ( $\mathrm{k}=4000 \mathrm{~A} / \mathrm{m}, \mathrm{c}=0.1$, $\left.M_{\text {sat }}=1.6 \mathrm{M} \mathrm{A} / \mathrm{m}, \alpha=0.003, a=2000 \mathrm{~A} / \mathrm{m}, a_{2}=3500 \mathrm{~A} / \mathrm{m}\right)$.

\section{Implementation in HDLs: SystemC and VHDL-AMS}

The model has been implemented in SystemC [3] and VHDL-AMS. The standard release of SystemC 2.01 is adequate in this case but an extended version with analogue support can be used as well. The following code shows part of the SystemC model.

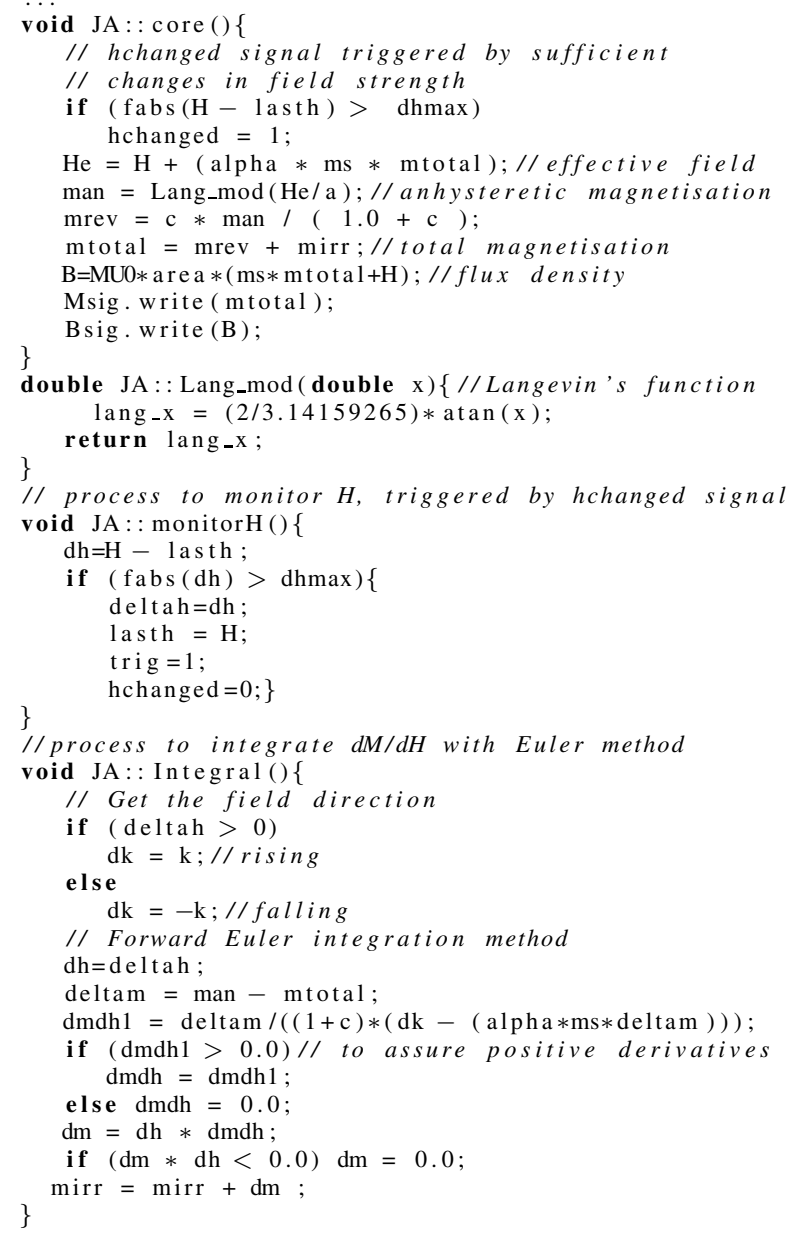

Process JA::core() is triggered on changes of the external magnetic field $H$. It calculates the anhysterestic from the modified Langevin's function (JA::Lang_mod()) [5] as well as the reversible and total magnetisation. JA::core() triggers the processes JA::monitorH() and JA::Integral() if an update of the magnetisation slope is necessary. The timeless approach to the slope discretisation avoids using time as the independent variable and the integral is calculated using increments of the magnetic field $H$ rather than time steps. For generality, a triangular waveform is used in a DC sweep, i.e. timeless simulations. The simulated $B H$ curve with non-biased minor loops is shown in Fig.1.

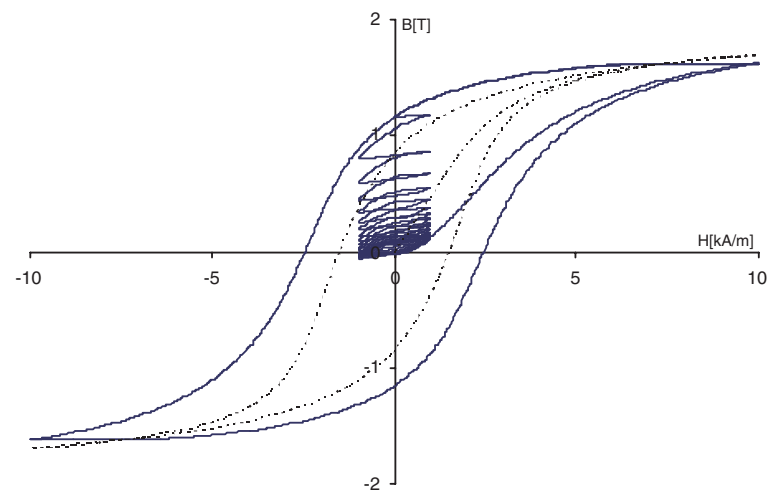

Figure 1. SystemC BH simulation.

\section{Conclusion}

This paper describes a numerically reliable, mixedphysical domain HDL implementation of the Jiles-Atherton model of ferromagnetic core hysteresis. The model was implemented in SystemC and VHDL-AMS. We have taken advantage of the HDL modeling potential and proposed a timeless discretisation technique for the magnetisation slope. This is the main contribution of the paper. Our implementation overcomes some reported problems that have occurred in other implementations, namely long simulation times, non-convergence and numerical instability.

\section{References}

[1] A. Brown, J. Ross, and K. Nichols. Time-Domain Simulation of Mixed Nonlinear Magnetic and Electronic Systems. IEEE Transactions on Magnetics, 37(1):522-532, January 2001.

[2] D. Jiles and D. Atherton. Theory of Ferromagnetic Hysteresis. Journal of Applied Physics, 55(6):2115-2120, 15 March 1984.

[3] Open SystemC Initiative OSCI, www.systemc.org. SystemC Language Reference Manual, 2003.

[4] P. Wilson and T. Kazmierski. A Novel Approach to MixedDomain Behavioral Modeling of Ferromagnetic hysteresis in VHDL-AMS. In Forum on Specification and Design Langauges, Lille-France, 13-17 September 2004.

[5] P. Wilson, J. Ross, A. Brown, T. Kazmierski, and J. Baranowski. Efficient Mixed-Domain Behavioural Modelling of Ferromagnetic Hysteresis Implemented in VHDL-AMS. In DATE, pages 742-743, Paris, France, February 2004. 